\title{
Time-staggered inhibition of JNK effectively sensitizes chemoresistant ovarian cancer cells to cisplatin and paclitaxel
}

\author{
MANABU SEINO ${ }^{1,2^{*}}$, MASASHI OKADA ${ }^{1 *}$, HIROTSUGU SAKAKI ${ }^{1,2}$, HIROYUKI TAKEDA $^{1,3}$, \\ HIKARU WATARAI ${ }^{1,4}$, SHUHEI SUZUKI ${ }^{1,3}$, SHIZUKA SEINO ${ }^{1,5}$, KENTA KURAMOTO ${ }^{1}$, \\ TSUYOSHI OHTA ${ }^{2}$, SATORU NAGASE ${ }^{2}$, HIROHISA KURACHI ${ }^{2,6}$ and CHIFUMI KITANAKA ${ }^{1,5}$ \\ Departments of ${ }^{1}$ Molecular Cancer Science, ${ }^{2}$ Obstetrics and Gynecology, ${ }^{3}$ Clinical Oncology and \\ ${ }^{4}$ The Second Department of Surgery, Yamagata University School of Medicine; ${ }^{5}$ Research Institute for the \\ Promotion of Medical Sciences, Yamagata University Faculty of Medicine, Yamagata 990-9585; \\ ${ }^{6}$ Osaka Medical Center and Research Institute for Maternal and Child Health, Osaka 594-1101, Japan
}

Received August 6, 2015; Accepted September 26, 2015

DOI: $10.3892 /$ or.2015.4377

\begin{abstract}
Ovarian cancer is the most lethal gynecological malignancy, for which platinum- and taxane-based chemotherapy plays a major role. Chemoresistance of ovarian cancer poses a major obstacle to the successful management of this devastating disease; however, effective measures to overcome platinum and taxane resistance are yet to be established. In the present study, while investigating the mechanism underlying the chemoresistance of ovarian cancer, we found that JNK may have a key role in the resistance of ovarian cancer cells to cisplatin and paclitaxel. Importantly, whereas simultaneous application of a JNK inhibitor and either of the chemotherapeutic agents had contrasting effects for cisplatin (enhanced cytotoxicity) and paclitaxel (decreased cytotoxicity), JNK inhibitor treatment prior to chemotherapeutic agent application invariably enhanced the cytotoxicity of both drugs, suggesting that the basal JNK activity is commonly involved in the chemoresistance of ovarian cancer cells to cisplatin and paclitaxel in contrast to drug-induced JNK activity which may have different roles for these two drugs. Furthermore, we confirmed using non-transformed human and rodent fibroblasts that sequential application of the JNK inhibitor and the chemotherapeutic agents did not augment their toxicity. Thus, our findings highlight for the first time the possible
\end{abstract}

Correspondence to: Professor Chifumi Kitanaka, Department of Molecular Cancer Science, Yamagata University School of Medicine, 2-2-2 Iida-nishi, Yamagata 990-9585, Japan

E-mail: ckitanak@med.id.yamagata-u.ac.jp

${ }^{*}$ Contributed equally

Key words: ovarian cancer, combination chemotherapy, JNK, pretreatment, co-treatment differential roles of the basal and induced JNK activities in the chemoresistance of ovarian cancer cells and also suggest that time-staggered JNK inhibition may be a rational and promising strategy to overcome the resistance of ovarian cancer to platinum- and taxane-based chemotherapy.

\section{Introduction}

Epithelial ovarian cancer, being the fourth leading cause of female cancer-related death in the developed world, is one of the most common and lethal gynecologic malignancies (1). The majority of ovarian cancer patients are diagnosed at advanced stages and undergo maximal cytoreductive surgery followed by a program of chemotherapy with a platinum agent and paclitaxel (2). While response to initial chemotherapy is remarkable in most cases, a significant proportion of ovarian cancers are originally resistant to platinum- and taxane-based chemotherapy, and even those ovarian cancers which respond well to the initial chemotherapy eventually recur with a high probability, most likely with increased chemoresistance $(1,2)$. Such intrinsic and acquired chemoresistance makes the management of ovarian cancer difficult and is therefore a contributing factor to the dismal prognosis of ovarian cancer. Apparently, elucidation of the underlying mechanism and development of novel measures to overcome the chemoresistance of ovarian cancer are direly needed to improve its prognosis. To date, extensive studies have identified a plethora of genes and molecular pathways implicated in the chemoresistance of ovarian cancer $(3,4)$; however, such information has not yet been fully translated into specific, effective measures to overcome the chemoresistance of ovarian cancer. Here in the present study, we investigated the molecular pathways responsible for the resistance of chemoresistant ovarian cancer cells. Our data suggest that JNK pathway activation plays a significant role in the chemoresistance of ovarian cancer cells and that JNK inhibition prior to the application of paclitaxel or cisplatin effectively sensitizes chemoresistant ovarian cancer cells to these chemotherapeutic agents. 


\section{Materials and methods}

Reagents and antibodies. SP600125 was purchased from Calbiochem (La Jolla, CA, USA) and dissolved in dimethylsulfoxide (DMSO) to prepare a $50 \mathrm{mM}$ stock solution. Cisplatin and paclitaxel were purchased from Sigma-Aldrich (St. Louis, MO, USA) and Wako Pure Chemical Industries, Ltd. (Osaka, Japan), respectively, and were dissolved in DMSO to prepare $100 \mathrm{mM}$ and $1 \mathrm{mM}$ stock solutions, respectively. Anti-c-Jun (\#9165), anti-phospho-c-Jun (\#9261) and anti-phospho-JNK (\#9251) antibodies were purchased from Cell Signaling Technology, Inc. (Beverly, MA, USA). Anti-JNK1 (sc-474) and anti-JNK2 (sc-7345) antibodies were purchased from Santa Cruz Biotechnology, Inc. (Santa Cruz, CA, USA), whereas anti- $\beta$-actin (A1978) was from Sigma-Aldrich.

Cell culture. Human ovarian cancer cell lines, TOV21-G and SKOV-3, were purchased from the American Type Culture Collection (ATCC, Manassas, VA, USA). A2780 was a kind gift from Dr T. Tsuruo at the Institute of Molecular and Cellular Biosciences, University of Tokyo, Japan and Drs R.F. Ozols and T.C. Hamilton at the National Institutes of Health, USA (5). A2780CP was kindly provided by the Department of Obstetrics and Gynecology, Osaka University, Japan. SKOV-3ip1 was a kind gift from Dr M.C. Hung at MD Anderson Cancer Center, University of Texas, USA (6). RMG-1 was kindly provided by Dr S. Nozawa and Dr D. Aoki at Keio University, Japan (7). TOV-21G, SKOV-3 and SKOV-3ipl cell lines were maintained in M199:105 medium, a 1:1 mixture of M199 and MCDB105 media supplemented with $10 \%$ (for SKOV-3 and SKOV-3ip1) or $15 \%$ (for TOV-21G) fetal bovine serum (FBS; Sigma) $(8,9)$. RMG-1, A2780 and A2780CP cell lines were maintained in DMEM/F12 medium supplemented with 10\% FBS $(10,11)$. Normal human IMR90 fetal lung fibroblasts, NIH3T3 mouse fibroblasts and Rat1 rat fibroblasts were obtained from ATCC and maintained in DMEM supplemented with $10 \%$ FBS. The culture medium was also supplemented with $100 \mathrm{U} / \mathrm{ml}$ penicillin and $100 \mu \mathrm{g} / \mathrm{ml}$ streptomycin. The culture medium was changed every 3 days. The authenticity of A2780CP, RMG-1, and SKOV-3 cells was verified by genotyping of short tandem repeat (STR) loci (BEX Co., Ltd., Tokyo, Japan) followed by comparison with the ATCC STR database for human cell lines. All IMR90 experiments were performed using low passage number $(<8)$ cells.

Cell viability assays. Viable and dead cells were identified by their ability and inability to exclude vital dyes, respectively (12). In brief, cells were stained with $0.2 \%$ trypan blue, and the numbers of viable and dead cells were determined using a hemocytometer. Cell viability (\%) was defined as $100 \times$ [number of viable cells/(number of viable + dead cells)], whereas the percentage of dead cells was defined as $100 \mathrm{x}$ [number of dead cells/(number of viable + dead cells)] To determine the $\mathrm{IC}_{50}$ values of cisplatin and paclitaxel for the ovarian cancer cell lines used in the present study, we treated the cells with varying concentrations of cisplatin or paclitaxel for 3 days and then determined their viability. The $\mathrm{IC}_{50}$ values were calculated using the following formula (13): $\mathrm{IC}_{50}=10^{[\log (\mathrm{A} / \mathrm{B}) \times(50-\mathrm{C})] /(\mathrm{D}-\mathrm{C})+\log (\mathrm{B})]}$ where $\mathrm{A}$ and $\mathrm{B}$ are the corresponding concentrations of the test drug directly above and

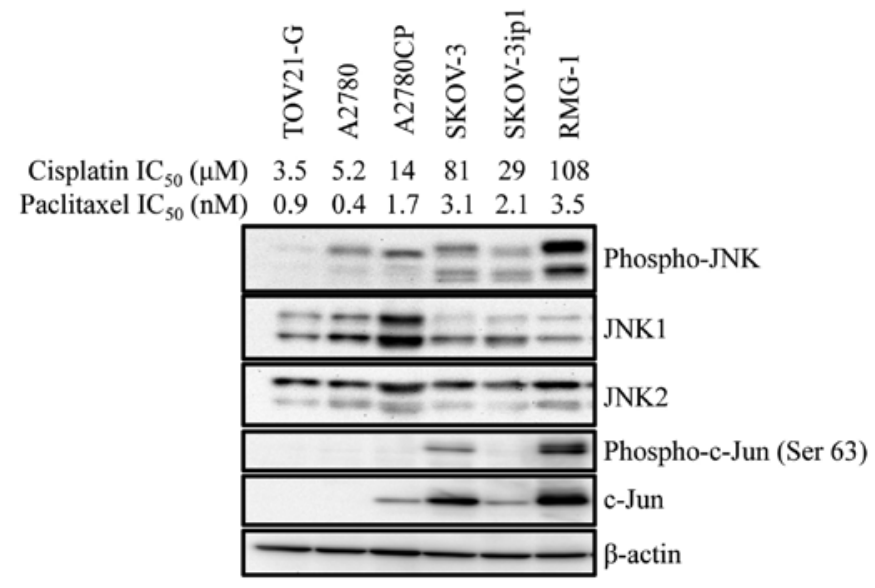

Figure 1. JNK pathway activity is increased in ovarian cancer cell lines resistant to cisplatin and paclitaxel. The indicated ovarian cancer cell lines were examined for the expression of JNK pathway proteins by immunoblot analysis. The $\mathrm{IC}_{50}$ values of cisplatin and paclitaxel for the cell lines were calculated as described in Materials and methods.

below 50\% inhibition, respectively, and $\mathrm{C}$ and $\mathrm{D}$ are the percentage of inhibition directly below and above 50\% inhibition, respectively.

Immunoblot analysis. Immunoblot analysis was conducted as described previously (14). In brief, cells were washed with ice-cold PBS and lysed in RIPA buffer [10 $\mathrm{mM}$ Tris- $\mathrm{HCl}$ ( $\mathrm{pH} 7.4$ ), $0.1 \%$ SDS, $0.1 \%$ sodium deoxycholate, $1 \%$ NP-40, $150 \mathrm{mM} \mathrm{NaCl}, 1 \mathrm{mM}$ EDTA, $1.5 \mathrm{mM} \mathrm{Na}_{3} \mathrm{VO}_{4}$, $10 \mathrm{mM} \mathrm{NaF}, 10 \mathrm{mM}$ sodium pyrophosphate, $10 \mathrm{mM}$ sodium $\beta$-glycerophosphate and $1 \%$ protease inhibitor cocktail set III (Sigma-Aldrich)]. After centrifugation for $10 \mathrm{~min}$ at $14,000 \mathrm{x} \mathrm{g}$ at $4^{\circ} \mathrm{C}$, the supernatants were recovered as the cell lysates, and the protein concentration of the cell lysates was determined using a BCA protein assay kit (Pierce Biotechnology, Inc., Rockford, IL, USA). Cell lysates containing equal amounts of protein were separated by SDS-PAGE and transferred to a polyvinylidene difluoride membrane. The membrane was probed with a primary antibody and then with an appropriate HRP-conjugated secondary antibody according to the protocol recommended by the manufacturer of each antibody. Immunoreactive bands were visualized using Immobilon Western Chemiluminescent HRP Substrate (Millipore, Billerica, MA, USA).

Colony formation assay. Colony formation assay was performed as described previously $(15,16)$. In brief, cells were seeded at a low, colony-forming density $\left(1 \times 10^{3}\right.$ cells $/ 60-\mathrm{mm}$ dish) and cultured for $\sim 2$ weeks. The cells were then fixed with formaldehyde $(4 \% \mathrm{v} / \mathrm{v})$, followed by staining with crystal violet $(0.1 \% \mathrm{w} / \mathrm{v})$. Colonies [consisting of $\geq 50$ cells derived from a single cell (progenies)] were counted using a microscope.

Statistical analysis. Results are expressed as the means \pm standard deviations (SD), and differences were compared using the two-tailed Student's t-test. $\mathrm{P}<0.05$ was considered to indicate a statistically significant difference and is indicated with asterisks in the figures. 
A

SP600125

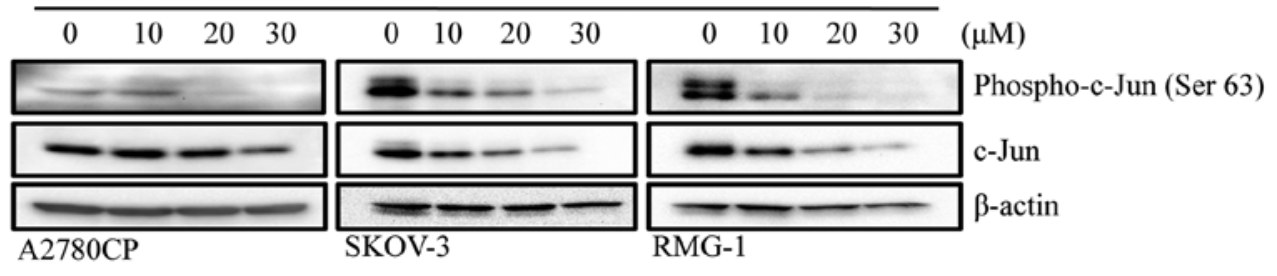

B

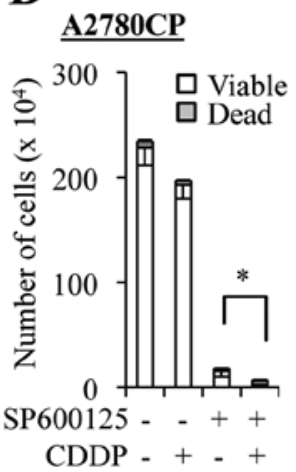

SKOV-3

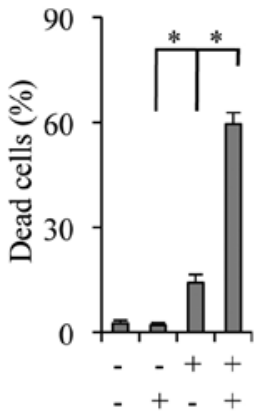

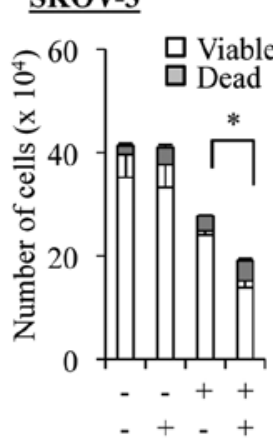

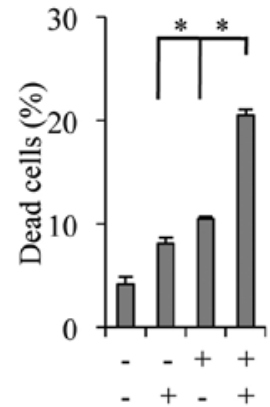

RMG-1

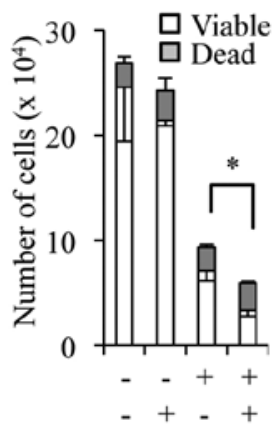

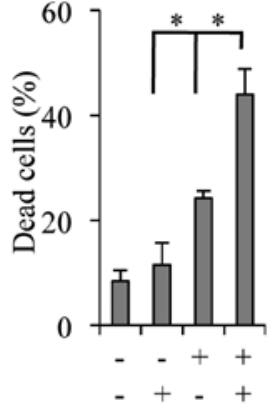

Figure 2. The growth inhibitory effect of cisplatin on ovarian cancer cells is enhanced in the presence of SP600125. (A) A2780CP, SKOV-3 and RMG-1 cells treated with the indicated concentrations of SP600125 for 3 days were subjected to immunoblot analysis of the indicated proteins. (B) A2780CP, SKOV-3 and RMG-1 cells were treated with cisplatin (CDDP, $50 \mu \mathrm{M})$ for 3 days in the absence or presence of SP600125 (20 $\mu \mathrm{M})$. Then the numbers of viable and dead cells (left panels) as well as the percentage of dead cells (right panels) were determined. Values in the graphs represent the means + SD (for the number and proportion of dead cells) and - SD (for the number of viable cells) of three independent experiments. ${ }^{*} \mathrm{P}<0.05$.

\section{Results}

Increased JNK pathway activity in human ovarian cancer cell lines resistant to cisplatin and paclitaxel. We previously reported that the expression of a dominant-negative c-Jun mutant sensitizes human ovarian cancer cell lines (A2780 and Caov-3) to cisplatin (17), which prompted us to hypothesize that the JNK pathway may have a role in the development of chemoresistance in ovarian cancer cells. As an initial approach to test this idea, we first examined the basal JNK activity in human ovarian cancer cell lines with various sensitivity/resistance to cisplatin. The results indicated that cisplatin-resistant cell lines tended to have higher basal JNK activity. Intriguingly, we also noticed at the same time that there was some parallelism between the sensitivity/resistance to cisplatin and paclitaxel. For instance, A2780CP, a cisplatin-resistant subline of A2780, was more resistant than the original A2780 cell line not only to cisplatin but also to paclitaxel (Fig. 1). Thus, the findings suggested that the basal JNK activity may be associated with cisplatin- and paclitaxel-resistance of human ovarian cancer cell lines.

Enhanced anticancer effects of cisplatin in ovarian cancer cells in the presence of a pharmacological JNK inhibitor SP600125. We next examined whether JNK is involved in the cisplatin resistance of the ovarian cancer cell lines. Since our previous study using a genetic approach showed that constitutive expression of a dominant-negative c-Jun mutant sensitized A2780 and Caov-3 cells to cisplatin (17), we wished to investigate in this particular study whether direct inhibition of JNK through a pharmacological approach could overcome the cisplatin-resistance of ovarian cancer cells, and that, of ovarian cancer cell lines other than A2780 and Caov-3. We therefore used SP600125, the most widely used pharmacological inhibitor of JNK (18), and tested its effect in three cisplatin-resistant ovarian cancer cell lines, A2780CP, SKOV-3, and RMG-1. Since SP600125 effectively inhibited the JNK activity at $20 \mu \mathrm{M}$ in all three cell lines as indicated by the reduced phosphorylation of c-Jun at the JNK phosphorylation site (Fig. 2A), we treated the cells with SP600125 at $20 \mu \mathrm{M}$, alone or in combination with cisplatin $(50 \mu \mathrm{M})$, and examined the effect of the drug treatment on their growth and viability (Fig. 2B). The results indicated that treatment with SP600125 alone caused a substantial inhibition of cell growth as well as a modest increase in the proportion of dead cells in the three cell lines. On the other hand, treatment of the cells with cisplatin alone affected their growth and viability only marginally, as expected from their inherent resistance to cisplatin. However, when the cells were treated with cisplatin in the presence of SP600125, the combination treatment caused a marked decrease in the number of viable cells accompanied by the corresponding increase in the proportion of dead cells, compared to treatment with either SP600125 or cisplatin alone. These findings extended our previous findings and suggested that the role of JNK in cisplatin resistance may be shared by different human ovarian cancer cell lines beyond A2780 and Caov-3, and that JNK could be a druggable target to overcome cisplatin resistance of ovarian cancer cells.

Diminished anticancer effects of paclitaxel in ovarian cancer cells in the presence of SP600125. Given our earlier observation in this study that the ovarian cancer cell lines with increased basal JNK activity were resistant not only to cisplatin but also to paclitaxel (Fig. 1), we next asked whether 

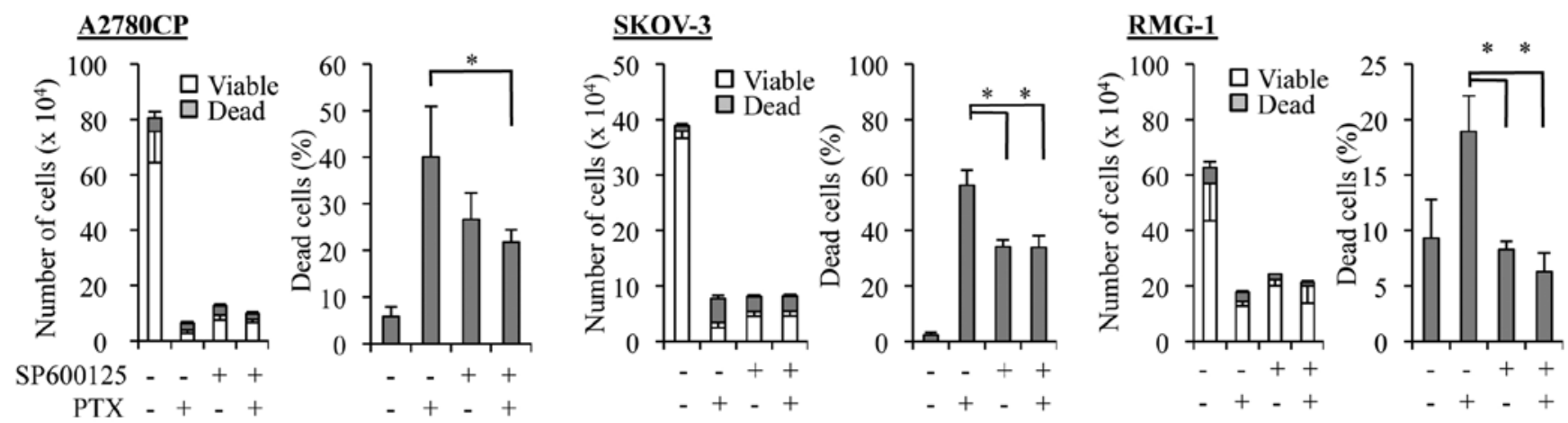

Figure 3. SP600125 co-treatment inhibits the cytotoxic effect of paclitaxel on ovarian cancer cells. (A) A2780CP, SKOV-3 and RMG-1 cells treated with paclitaxel (PTX, $5 \mathrm{nM}$ ) for 3 days in the absence or presence of SP600125 (20 $\mu \mathrm{M})$. The numbers of viable and dead cells (left panels) as well as the percentage of dead cells (right panels) were determined. Values in the graphs represent the means + SD (for the number and proportion of dead cells) and - SD (for the number of viable cells) of three independent experiments. ${ }^{*} \mathrm{P}<0.05$.
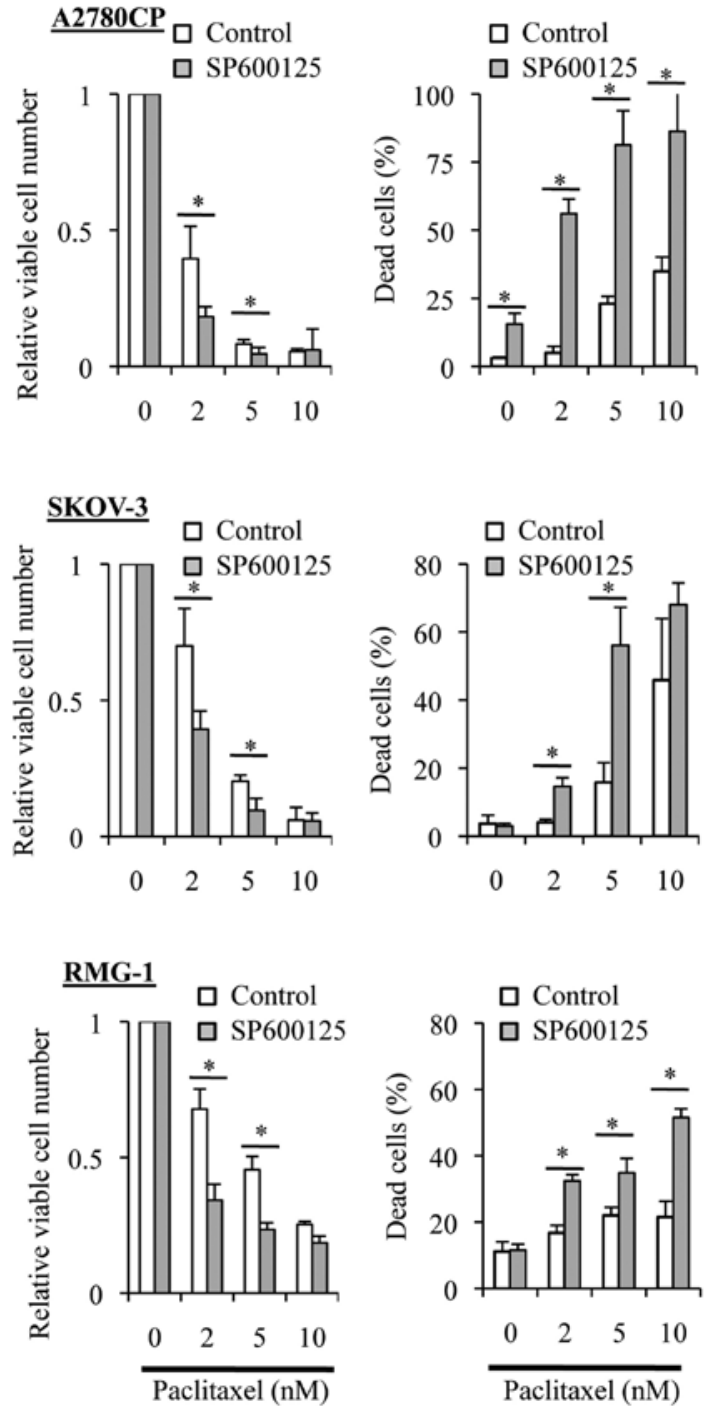

Figure 4. Pretreatment with SP600125 enhances the cytotoxic effect of paclitaxel on ovarian cancer cells. A2780CP, SKOV-3 and RMG-1 cells pretreated without (white bars) or with (gray bars) SP600125 $(20 \mu \mathrm{M})$ for 3 days were subsequently treated with the indicated concentrations of paclitaxel for 3 days, after which the cell viability was determined. The number of viable cells relative to the controls (equals the numbers of viable cells after treatment 'without' paclitaxel in the presence and absence of SP600125 pretreatment) and the percentage of dead cells are shown in the left and right panels, respectively. Values in the graphs represent the means + SD of three independent experiments. ${ }^{*} \mathrm{P}<0.05$.
SP600125 treatment could enhance the anticancer effects of paclitaxel similarly to those of cisplatin. However, we found that the growth inhibitory effect of paclitaxel was not at all enhanced in the presence of SP600125. On the contrary, SP600125 even compromised the paclitaxel effect on ovarian cancer cells. Indeed, when the paclitaxel-resistant cell lines were treated with paclitaxel at a sufficiently high concentration $(5 \mathrm{nM})$ to cause growth inhibition in the presence and absence of SP600125, the number of viable cells increased whereas that of dead cells decreased in the presence of SP600125 as compared with its absence (Fig. 3).

Treatment with SP600125 prior to paclitaxel enhances the anticancer effects of paclitaxel in ovarian cancer cells. A number of previous studies showed that paclitaxel treatment of cancer cells, including ovarian cancer cells, caused JNK activation and that the JNK activation was required for paclitaxel-induced cell death (19-23). Whereas these observations appear to imply that the 'induced' JNK activation plays an essential role in cell death caused by paclitaxel treatment, which is quite consistent with the results of the present study (Fig. 3), the role of the 'basal' JNK activity in cells treated with paclitaxel remains unknown. To determine the role of the basal JNK activity in the sensitivity/resistance of ovarian cancer cells to paclitaxel, we tested the effect of time-staggered, sequential application of SP600125 and paclitaxel in the present study. To specifically inhibit the basal JNK activity without interfering with paclitaxel-induced activation of JNK, the ovarian cancer cell lines were first treated for 3 days with SP600125, which is a reversible inhibitor of JNK, followed by paclitaxel treatment in the entire absence of SP600125. Strikingly, in sharp contrast to the results obtained by treating cells simultaneously with SP600125 and paclitaxel (i.e., SP600125 co-treatment), prior treatment with SP600125 (i.e., SP600125 pretreatment) significantly enhanced the growth inhibitory and cell death-inducing effects of paclitaxel over a range of concentration (Fig. 4). These observations suggested that specific inhibition of the basal JNK activity may effectively sensitize ovarian cancer cells to paclitaxel.

Treatment with SP600125 prior to cisplatin enhances the anticancer effects of cisplatin in ovarian cancer cells. Our 

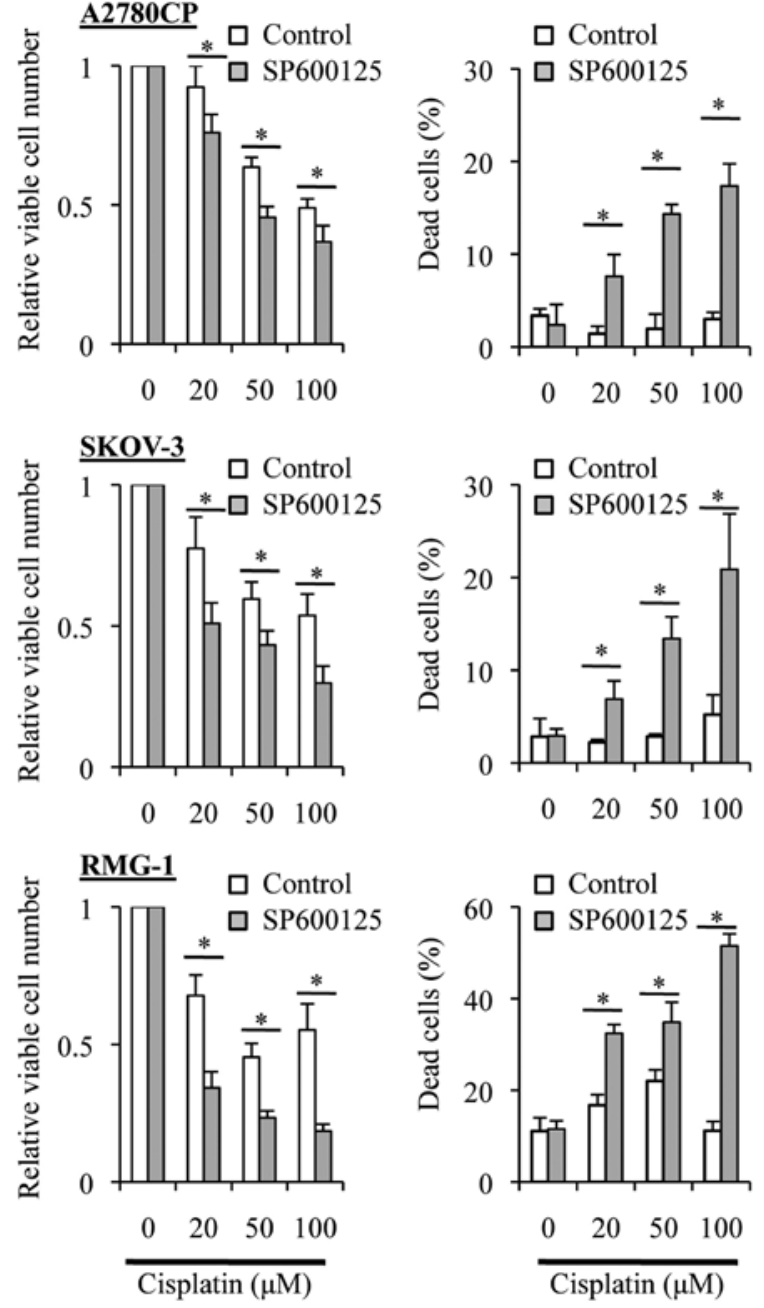

Figure 5. Pretreatment with SP600125 enhances the cytotoxic effect of cisplatin on ovarian cancer cells. A2780CP, SKOV-3 and RMG-1 cells pretreated without (white bars) or with (gray bars) SP600125 $(20 \mu \mathrm{M})$ for 3 days were subsequently treated with the indicated concentrations of cisplatin for 3 days, after which the cell viability was determined. The number of viable cells relative to the controls (equals the numbers of viable cells after treatment 'without' cisplatin in the presence and absence of SP600125 pretreatment) and the percentage of dead cells are shown in the left and right panels, respectively. Values in the graphs represent the means + SD of three independent experiments. ${ }^{*} \mathrm{P}<0.05$.

earlier observations demonstrated that cisplatin was different from paclitaxel in that SP600125 'co-treatment' sensitized the ovarian cancer cell lines to cisplatin (Fig. 2) but not to paclitaxel (Fig. 3). We then asked whether or not SP600125 'pretreatment' sensitizes the ovarian cancer cell lines to cisplatin as it did to paclitaxel (Fig. 4). When the ovarian cancer cell lines were treated with varying concentrations of cisplatin in the absence of SP600125 after being exposed or not to SP600125 for 3 days, we found that the growth inhibitory and cell death-inducing effects of cisplatin were significantly enhanced by the SP600125 pretreatment (Fig. 5). These results, together with those obtained from the paclitaxel experiments (Fig. 4), suggested that the basal JNK activity may be commonly involved in the resistance of the ovarian cancer cell lines to cisplatin and paclitaxel.

SP600125 pretreatment effectively synergizes with both cisplatin and paclitaxel to suppress the clonogenic survival of ovarian cancer cells. The results of the present study suggest that SP600125 pretreatment effectively enhances the anticancer effects of cisplatin and paclitaxel. However, we assessed the anticancer effects of the drugs so far by determining the number and proportion of viable and dead cells at a relatively early time point, namely, 3 days after the application of the drugs. It was therefore possible, for instance, that SP600125 pretreatment was simply advancing the time-kinetics of cell death that would eventually occur, making cells destined to die, die earlier. To exclude such a possibility and determine the therapeutic significance of SP600125 pretreatment (24), we examined the impact of SP600125 pretreatment on the clonogenic survival of ovarian cancer cells. To this end, we first exposed the ovarian cancer cell lines to SP600125 and paclitaxel alone and in combinations and subjected them to the colony formation assay. For combination treatments, cells were either exposed to SP600125 and paclitaxel simultaneously (co-treatment, the SP + PTX protocol), or to SP600125 followed by paclitaxel (pretreatment, the SP $\rightarrow$ PTX protocol). The results indicated that, in all three cell lines examined, whereas the co-treatment protocol was at best as effective as paclitaxel alone in inhibiting the clonogenic survival of the cells, the pretreatment protocol was by far more effective than paclitaxel alone and the co-treatment protocol (Fig. 6). We then went on to conduct the same experiment with cisplatin. In line with our earlier results, the co-treatment protocol tended to be more effective than cisplatin alone. However, again, the pretreatment protocol was significantly more effective than the co-treatment protocol, just as with paclitaxel (Fig. 7). These findings suggested that pretreatment with SP600125 may be beneficial in augmenting the therapeutic efficacy of cisplatin and paclitaxel against ovarian cancer cells.

The SP600125 pretreatment protocol does not augment the cytotoxic effect of cisplatin and paclitaxel on non-transformed fibroblasts. We next asked whether the combination treatment protocols might increase the cytotoxic effects of cisplatin and paclitaxel on normal cells. To this end, we used non-transformed fibroblasts from human (IMR90), mouse (NIH3T3), and rat (Rat1), and tested the effects of cisplatin, paclitaxel and SP600125 alone or in combination. Cisplatin treatment $\leq 100 \mu \mathrm{M}$ alone did not appreciably reduce the viability (=100\% - \%dead cells) of these fibroblasts, and neither did paclitaxel alone even at $10 \mathrm{nM}$ (Fig. 8). SP600125 (20 $\mu \mathrm{M})$ alone occasionally reduced the viability of IMR90 cells significantly, which was nevertheless not reproducible (Fig. 8A and B). Importantly, the combination protocols (co-treatment and pretreatment protocols for both cisplatin and paclitaxel) reduced the viability of the cells significantly in none of the three nontransformed fibroblasts. Thus, the results suggested that the combination protocols could enhance the anticancer effects of the drugs without increasing their toxicity.

\section{Discussion}

Chemoresistance eventually develops in the majority of ovarian cancer cases during the clinical course and is therefore a major obstacle in realizing the long-term survival of patients with ovarian cancer. To search for strategies to overcome the resistance of ovarian cancer to platinum- and taxane-based 

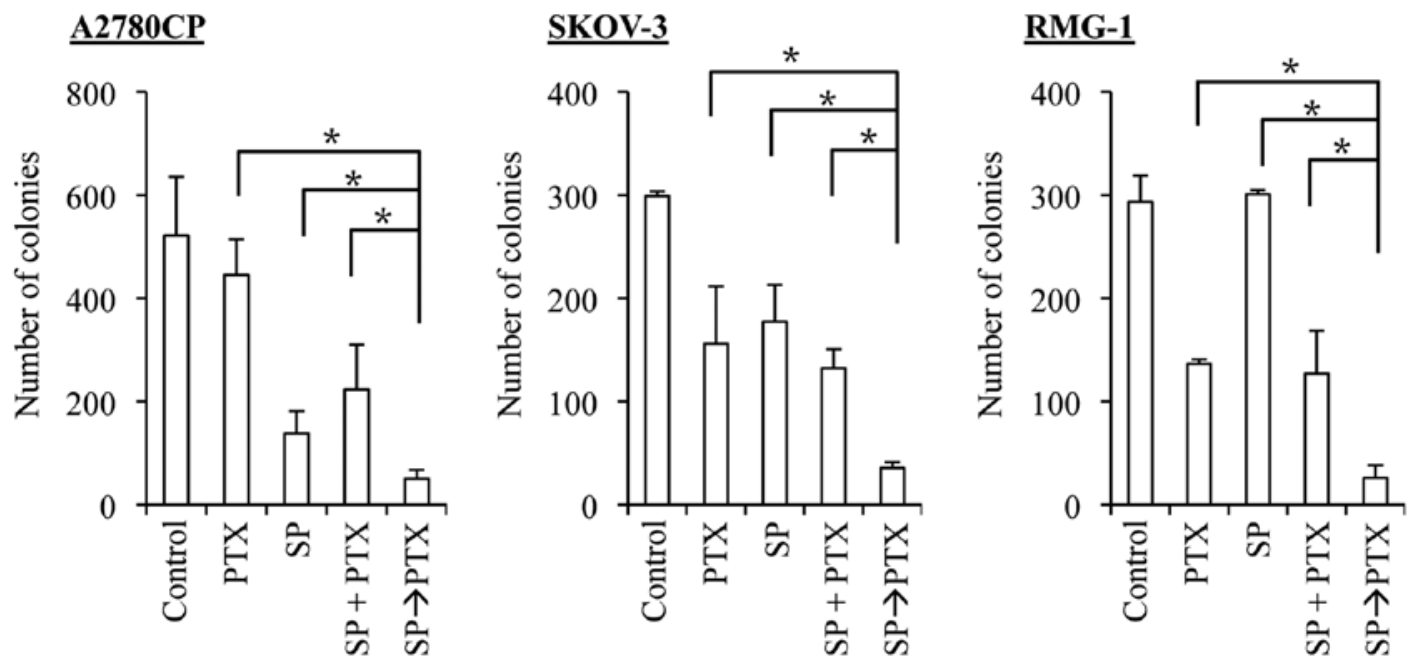

Figure 6. Pretreatment with SP600125 enhances the inhibitory effect of paclitaxel on the clonogenic survival of ovarian cancer cells. A2780CP, SKOV-3 and RMG-1 cells pretreated without or with SP600125 $(20 \mu \mathrm{M})$ for 3 days were subsequently treated without or with paclitaxel (2 nM) for 3 days (control, PTX, $\mathrm{SP}$ and $\mathrm{SP} \rightarrow \mathrm{PTX})$. Alternatively, the cells were treated with paclitaxel $(2 \mathrm{nM})$ in the presence of SP600125 $(20 \mu \mathrm{M})$ for 3 days $(\mathrm{SP}+\mathrm{PTX})$. The cells were then subjected to colony formation assay in the absence of drugs. Values in the graphs represent the means $+\mathrm{SD}$ of three independent experiments. ${ }^{*} \mathrm{P}<0.05$.
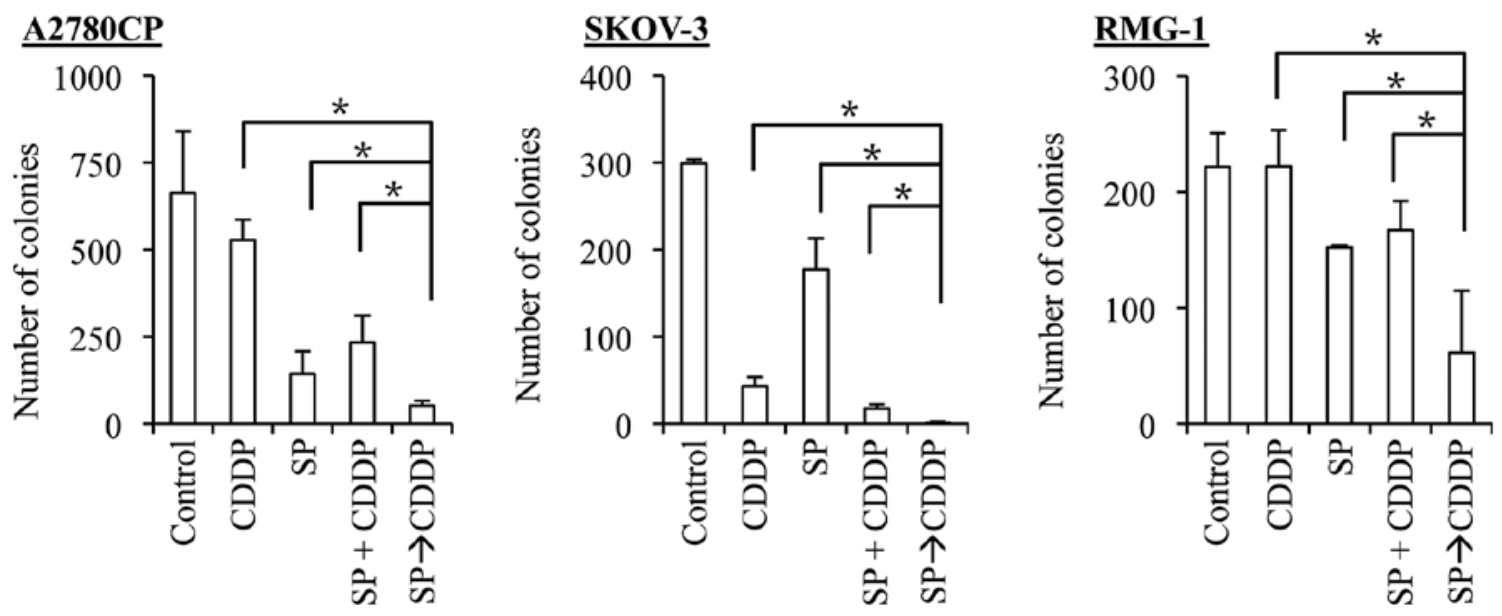

Figure 7. Pretreatment with SP600125 enhances the inhibitory effect of cisplatin on the clonogenic survival of ovarian cancer cells. A2780CP, SKOV-3 and RMG-1 cells pretreated without or with SP600125 $(20 \mu \mathrm{M})$ for 3 days were subsequently treated without or with cisplatin $(50 \mu \mathrm{M})$ for 3 days (control, CDDP, $\mathrm{SP}$ and $\mathrm{SP} \rightarrow \mathrm{CDDP})$. Alternatively, the cells were treated with cisplatin $(50 \mu \mathrm{M})$ in the presence of SP600125 $(20 \mu \mathrm{M})$ for 3 days (SP + CDDP). The cells were then subjected to colony formation assay in the absence of drugs. Values in the graphs represent the means $+\mathrm{SD}$ of three independent experiments. "P<0.05.

chemotherapy, we attempted in this study to identify molecules and/or pathways that may dictate the sensitivity/resistance of ovarian cancer cells to cisplatin and paclitaxel. As a result, we found in the present study that i) there is some parallelism between the sensitivity/resistance of each human ovarian cancer cell line to cisplatin and paclitaxel, ii) the JNK pathway activity (basal activity) is positively correlated with resistance to cisplatin and paclitaxel, iii) chemotherapeutic agent-induced activation of JNK is likely involved in the resistance and sensitivity to cisplatin and paclitaxel, respectively, iv) the basal JNK activity confers resistance to both cisplatin and paclitaxel and v) inhibition of the basal JNK activity prior to the application of cisplatin or paclitaxel effectively augments their ability to suppress the clonogenic survival of ovarian cancer cells without increasing their toxicity. These findings not only provide a useful clue to develop specific measures to sensitize ovarian cancer to platinum- and taxane-based chemotherapy but also shed insights into the mechanisms underlying the chemoresistance of ovarian cancer.

Previous studies have shown that treatment of cancer cell lines with cisplatin in vitro often leads to the development of selective resistance to cisplatin without cross-resistance to paclitaxel and vice versa, most likely because of their entirely distinct mechanisms of action $(25,26)$. On the other hand, we observed in the present study a parallelism between sensitivity/resistance to cisplatin and paclitaxel across human ovarian cancer cell lines maintained in a drug-naïve culture condition (A2780, TOV21-G, SKOV-3, SKOV-3ip1, RMG-1) and a subline established by exposing the parental cell lines to cisplatin but maintained in a drug-free culture condition (A2780CP). Collectively, these observations may imply that intrinsic resistance, which is inherent in cells irrespective of prior drug treatment, and acquired resistance, which is induced by drug treatment, involve different underlying mechanisms, 
A $\underline{\text { IMR90 }}$

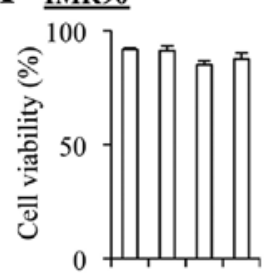

SP600125 - - + +

PTX - + - +

\section{B $\underline{\text { IMR90 }}$}

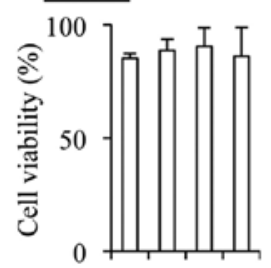

SP600125 - - + +

CDDP - + - +

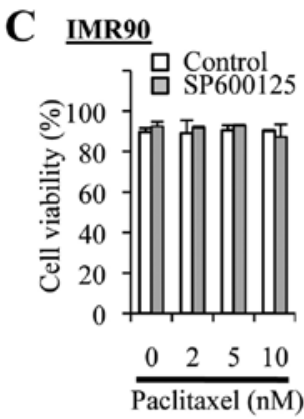

D

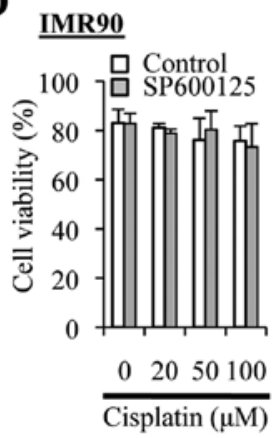

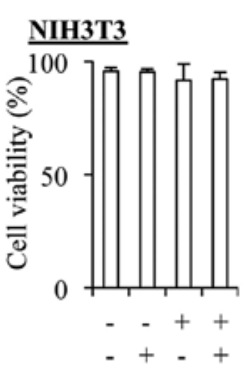
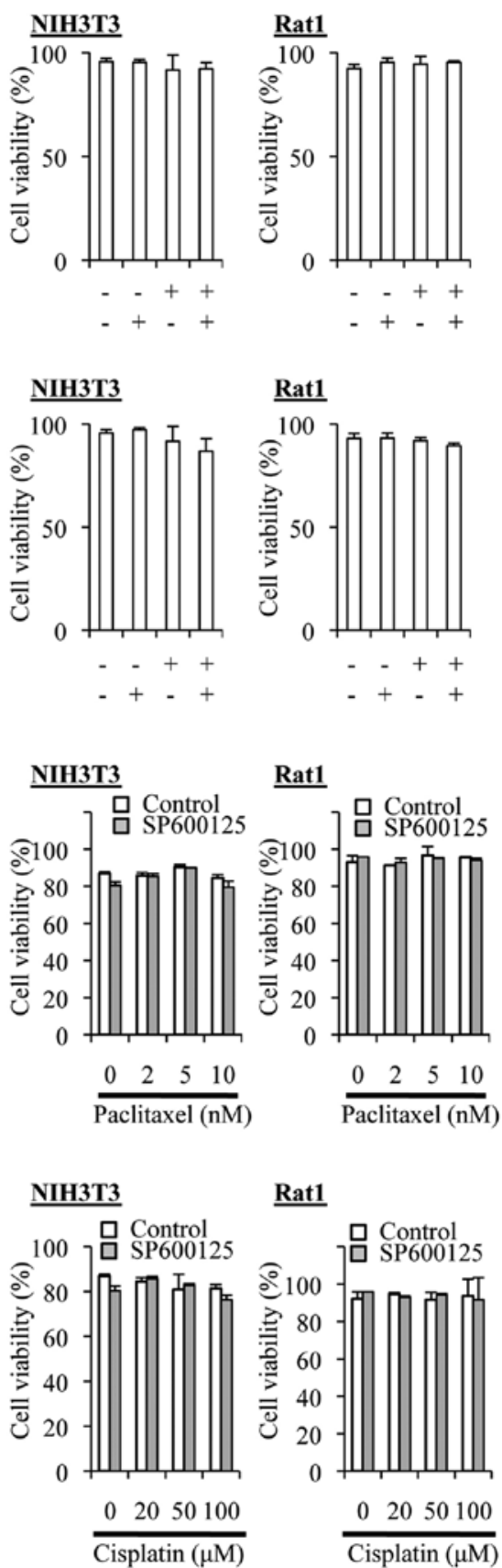

$\underline{\text { Rat1 }}$

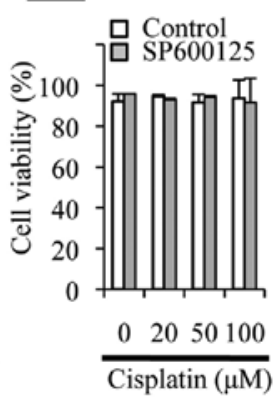

Figure 8. Pretreatment with SP600125 does not increase the cytotoxic effect of paclitaxel or cisplatin on nontransformed fibroblasts. (A and B) IMR90, NIH3T3 and Rat1 cells were treated with paclitaxel (A: PTX, $5 \mathrm{nM}$ ) or cisplatin (B: CDDP, $50 \mu \mathrm{M}$ ) for 3 days in the absence or presence of SP600125 $(20 \mu \mathrm{M})$, after which the cell viability was determined. (C and D) Cells pretreated without (white bars) or with (gray bars) SP600125 $(20 \mu \mathrm{M})$ for 3 days were subsequently treated with the indicated concentrations of paclitaxel (C) or cisplatin (D) for 3 days, after which the cell viability was determined. Values represent the means $+\mathrm{SD}$ of three independent experiments.

the latter being, as a natural consequence, more specific to the particular chemotherapeutic agents used to induce resistance. Quite importantly, we found that the basal JNK pathway activity was higher in cell lines that were inherently more resistant to both cisplatin and paclitaxel and that inhibition of the basal JNK activity prior to the application of these drugs sensitized ovarian cancer cells to both of them. These findings point to the intriguing possibility that JNK plays a key role in determining the intrinsic, basal resistance of ovarian cancer cells to platinum- and taxane-based chemotherapy, although the mechanism involved in the intrinsic, basal resistance could nevertheless be induced or augmented on occasions by treatment with chemotherapeutic agents, as is evident form the comparison of $\mathrm{A} 2780$ and $\mathrm{A} 2780 \mathrm{CP}$ in the present study. Intriguing enough, the JNK pathway has been demonstrated to be activated in a significant proportion of ovarian cancers (27,28). More importantly, active JNK expression level was higher in advanced stage (III and IV) cases than in early stage (I and II) cases and was inversely associated with the survival of ovarian cancer patients independently of the clinical stage (27), suggesting that the JNK pathway activity may increase with disease progression and have a negative impact on the clinical outcome of ovarian cancer patients. Our results, in conjunction with these previous observations, may give rise to a novel, provocative hypothesis that ovarian cancers become chemoresistant not simply because they are treated with chemotherapy but because they progress with time to more advanced disease with increased JNK activity in their natural clinical course.

Another important aspect of the present study is that we delineated and highlighted the distinct roles of JNK in the intrinsic resistance mechanism of ovarian cancer cells to cisplatin and paclitaxel in the untreated, steady state condition and in their acute response to these drugs. The roles of JNK in the acute response of cancer cells to cisplatin (17,29-33) and paclitaxel (19-23) have been well documented. In line with the previous reports demonstrating the critical role of JNK in the activation of the DNA repair mechanism after cisplatin treatment (17,29-33), cisplatin application along with the JNK inhibitor SP600125 resulted in enhanced anticancer effects compared with cisplatin application alone in the present study. In contrast, paclitaxel application along with SP600125 resulted in diminished anticancer effects, again in line with the reported role of JNK in mediating the death signal elicited by paclitaxel (19-23). Thus, the results of the present study further strengthen the idea that JNK has contrasting roles in the acute response of cancer cells to cisplatin and paclitaxel. On the other hand, the role of JNK in the mechanism(s) dictating the intrinsic sensitivity and resistance of cancer cells not yet exposed to these drugs has been poorly investigated and therefore remains totally obscure. Here in the present study, we provide evidence supporting the idea that the basal JNK activity plays a pivotal role in maintaining the intrinsic resistance of ovarian cancer cells to both cisplatin and paclitaxel. It still remains to be shown how JNK contributes to the intrinsic resistance of ovarian cancer cells to both cisplatin and paclitaxel, however, two major possibilities could be envisaged. The first possibility is that JNK is involved separately in each of the distinct mechanisms underlying cisplatin and paclitaxel resistance. For intrinsic resistance to cisplatin, it may not be difficult to assume that the basal JNK activity has a role not only in induced but also in the steady state DNA repair activity, conferring on cancer cells a higher capacity to repair DNA damages prior to cisplatin exposure. As for intrinsic paclitaxel resistance, it has been reported that cisplatin-resistant cancer cells that are also cross-resistant to palcitaxel often have upregulation of p-glycoprotein, which is considered to be responsible for paclitaxel resistance (34-39). Given the previous reports 
suggesting a role for JNK in the regulation of the MDR1 gene that encodes p-glycoprotein $(40,41)$, it is plausible to assume that p-glycoprotein is involved in the JNK-dependent paclitaxel resistance observed in this study. However, the results of our pilot experiment indicated that MDR1 expression was not necessarily decreased in the cell lines sensitized to paclitaxel by SP600125 treatment (data not shown), suggesting that at least MDR1 expression alone may not be accountable for the paclitaxel resistance. The second possibility is that JNK is involved in a mechanism common to the manifestation of the anticancer effects of cisplatin and paclitaxel, most likely in the cell death and cell cycle pathways. In this regard, in so far as we have examined, we have not yet identified cell death or cell cycle molecules whose expression reasonably changes upon SP600125 treatment. Apparently, future studies are warranted to elucidate the mechanism by which the basal JNK activity contributes to the intrinsic resistance of ovarian cancer cells to cisplatin and paclitaxel.

Finally and most importantly, we demonstrated in the present study that sequential, but not simultaneous, exposure to SP600125 and cisplatin/paclitaxel in this order effectively sensitizes ovarian cancer cells to these chemotherapeutic agents, which has led us to propose that time-staggered inhibition of JNK in combination with cisplatin and paclitaxel may be beneficial in the treatment of ovarian cancer. Recently, there has been a growing awareness that not only the combination and dosage of drugs, but also the timing, duration, and order of the drugs to be combined are key to successful combination treatment (42-44). Our study thus provides a good illustration of the idea, underscoring the importance of the order, timing, and duration of drug application in combination treatment. In the present study, the ovarian cancer cells were treated with the JNK inhibitor for 3 days before exposure to cisplatin or paclitaxel, which we consider was required and sufficient for rewiring of the JNK signaling network to mitigate their chemoresistance. It is also important to emphasize here that the sequential treatment protocols (SP600125 $\rightarrow$ cisplatin, SP600125 $\rightarrow$ paclitaxel) were no more toxic to non-transformed cells than treatment with cisplatin or paclitaxel alone, indicating that SP600125 treatment prior to the application of the chemotherapeutic agents is quite beneficial in widening their therapeutic window. All in all, our findings demonstrate for the first time that, while simultaneous treatment with a JNK inhibitor could even be hazardous because it may not be without the risk of reducing the efficacy of taxane-based chemotherapy, time-staggered inhibition of JNK in combination with platinum- and taxane-based chemotherapy is highly useful in enhancing the therapeutic effects of the chemotherapeutic agents. Furthermore, we recently demonstrated that the JNK activity is required for the maintenance of ovarian cancer stem cells and, although not in ovarian but in pancreatic cancer, that time-staggered inhibition of JNK effectively sensitizes cancer stem cells to chemotherapeutic agents $(11,12)$. These observations may make combination therapies involving JNK inhibitors all the more attractive as an approach to the treatment of ovarian cancer. Apparently, future preclinical studies are warranted to determine the therapeutic effects of combination treatment consisting of time-staggered JNK inhibition and platinum- and taxane-based chemotherapy, as a pilot to explore the clinical relevance of such combination treatment.

\section{Acknowledgements}

We thank Ms Eriko Watanabe and Ms Asuka Sugai for their technical and secretarial contributions to this study, respectively. The present study was supported by Grants-in-Aid for Scientific Research, for Challenging Exploratory Research, and for Young Scientists from the Ministry of Education, Culture, Sports, Science and Technology of Japan.

\section{References}

1. Jayson GC, Kohn EC, Kitchener HC and Ledermann JA: Ovarian cancer. Lancet 384: 1376-1388, 2014.

2. Bookman MA: The addition of new drugs to standard therapy in the first-line treatment of ovarian cancer. Ann Oncol 21 (Suppl 7): vii211-vii217, 2010.

3. Liu X, Gao Y, Lu Y, Zhang J, Li L and Yin F: Oncogenes associated with drug resistance in ovarian cancer. J Cancer Res Clin Oncol 141: 381-395, 2015 .

4. Yin F, Liu X, Li D, Wang Q,Zhang W and Li L: Tumor suppressor genes associated with drug resistance in ovarian cancer (Review). Oncol Rep 30: 3-10, 2013.

5. Hamilton TC, Winker MA, Louie KG, Batist G, Behrens BC, Tsuruo T, Grotzinger KR, McKoy WM, Young RC and Ozols RF: Augmentation of adriamycin, melphalan, and cisplatin cytotoxicity in drug-resistant and -sensitive human ovarian carcinoma cell lines by buthionine sulfoximine mediated glutathione depletion. Biochem Pharmacol 34: 2583-2586, 1985.

6. Yu D, Wolf JK, Scanlon M, Price JE and Hung MC: Enhanced c-erbB-2/neu expression in human ovarian cancer cells correlates with more severe malignancy that can be suppressed by E1A. Cancer Res 53: 891-898, 1993.

7. Suzuki N, Aoki D, Tamada Y, Susumu N, Orikawa K, Tsukazaki K, Sakayori M, Suzuki A, Fukuchi T, Mukai M, et al: HMOCC-1, a human monoclonal antibody that inhibits adhesion of ovarian cancer cells to human mesothelial cells. Gynecol Oncol 95: 290-298, 2004.

8. Liu Z, Yamanouchi K, Ohtao T, Matsumura S, Seino M, Shridhar V, Takahashi T, Takahashi K and Kurachi H: High levels of Wilms' tumor 1 (WT1) expression were associated with aggressive clinical features in ovarian cancer. Anticancer Res 34: 2331-2340, 2014.

9. Yamanouchi K, Ohta T, Liu Z, Oji Y, Sugiyama H, Shridhar V, Matsumura S, Takahashi T, Takahashi K and Kurachi H: The Wilms' tumor gene WT1 - 17AA/- KTS splice variant increases tumorigenic activity through up-regulation of vascular endothelial growth factor in an in vivo ovarian cancer model. Transl Oncol 7: 580-589, 2014.

10. Ohta T, Ohmichi M, Shibuya T, Takahashi T, Tsutsumi S, Takahashi K and Kurachi H: Gefitinib (ZD1839) increases the efficacy of cisplatin in ovarian cancer cells. Cancer Biol Ther 13: 408-416, 2012.

11. Seino M, Okada M, Shibuya K, Seino S, Suzuki S, Ohta T, Kurachi $\mathrm{H}$ and Kitanaka C: Requirement of JNK signaling for self-renewal and tumor-initiating capacity of ovarian cancer stem cells. Anticancer Res 34: 4723-4731, 2014.

12. Suzuki S, Okada M, Shibuya K, Seino M, Sato A, Takeda H, Seino S, Yoshioka T and Kitanaka C: JNK suppression of chemotherapeutic agents-induced ROS confers chemoresistance on pancreatic cancer stem cells. Oncotarget 6: 458-470, 2015.

13. Kiguchi K, Kubota T, Aoki D, Udagawa Y, Yamanouchi S, Saga M, Amemiya A, Sun FX, Nozawa S, Moossa AR, et al: A patient-like orthotopic implantation nude mouse model of highly metastatic human ovarian cancer. Clin Exp Metastasis 16: 751-756, 1998.

14. Okada M, Shibuya K, Sato A, Seino S, Suzuki S, Seino M and Kitanaka C: Targeting the K-Ras-JNK axis eliminates cancer stem-like cells and prevents pancreatic tumor formation. Oncotarget 5: 5100-5112, 2014.

15. Okada M, Sato A, Shibuya K, Watanabe E, Seino S, Suzuki S, Seino M, Narita Y, Shibui S, Kayama T, et al: JNK contributes to temozolomide resistance of stem-like glioblastoma cells via regulation of MGMT expression. Int J Oncol 44: 591-599, 2014.

16. Wang X, Song H, Yu Q, Liu Q, Wang L, Liu Z and Yu Z: Ad-p53 enhances the sensitivity of triple-negative breast cancer MDA-MB-468 cells to the EGFR inhibitor gefitinib. Oncol Rep 33: 526-532, 2015. 
17. Hayakawa J, Ohmichi M, Kurachi H, Ikegami H, Kimura A, Matsuoka T, Jikihara H, Mercola D and Murata Y: Inhibition of extracellular signal-regulated protein kinase or c-Jun N-terminal protein kinase cascade, differentially activated by cisplatin, sensitizes human ovarian cancer cell line. J Biol Chem 274: 31648-31654, 1999.

18. Bogoyevitch MA and Arthur PG: Inhibitors of c-Jun N-terminal kinases: JuNK no more? Biochim Biophys Acta 1784: 76-93, 2008.

19. Kolomeichuk SN, Terrano DT, Lyle CS, Sabapathy K and Chambers TC: Distinct signaling pathways of microtubule inhibitors - vinblastine and Taxol induce JNK-dependent cell death but through AP-1-dependent and AP-1-independent mechanisms, respectively. FEBS J 275: 1889-1899, 2008.

20. Lee LF, Li G, Templeton DJ and Ting JP: Paclitaxel (Taxol)induced gene expression and cell death are both mediated by the activation of c-Jun NH2-terminal kinase (JNK/SAPK). J Biol Chem 273: 28253-28260, 1998.

21. Selimovic D, Hassan M, Haikel Y and Hengge UR: Taxol-induced mitochondrial stress in melanoma cells is mediated by activation of c-Jun N-terminal kinase (JNK) and p38 pathways via uncoupling protein 2. Cell Signal 20: 311-322, 2008.

22. Sunters A, Madureira PA, Pomeranz KM, Aubert M, Brosens JJ, Cook SJ, Burgering BM, Coombes RC and Lam EW: Paclitaxel-induced nuclear translocation of FOXO3a in breast cancer cells is mediated by c-Jun NH2-terminal kinase and Akt. Cancer Res 66: 212-220, 2006.

23. Wang TH, Popp DM, Wang HS, Saitoh M, Mural JG, Henley DC Ichijo $\mathrm{H}$ and Wimalasena J: Microtubule dysfunction induced by paclitaxel initiates apoptosis through both c-Jun N-terminal kinase (JNK)-dependent and -independent pathways in ovarian cancer cells. J Biol Chem 274: 8208-8216, 1999.

24. Abend M: Reasons to reconsider the significance of apoptosis for cancer therapy. Int J Radiat Biol 79: 927-941, 2003.

25. Stordal B and Davey R: A systematic review of genes involved in the inverse resistance relationship between cisplatin and paclitaxel chemotherapy: Role of BRCA1. Curr Cancer Drug Targets 9: 354-365, 2009 .

26. Stordal B, Pavlakis N and Davey R: A systematic review of platinum and taxane resistance from bench to clinic: An inverse relationship. Cancer Treat Rev 33: 688-703, 2007.

27. Eckhoff K, Flurschütz R, Trillsch F, Mahner S, Jänicke F and Milde-Langosch K: The prognostic significance of Jun transcription factors in ovarian cancer. J Cancer Res Clin Oncol 139: 1673-1680, 2013

28. Vivas-Mejia P, Benito JM, Fernandez A, Han HD, Mangala L, Rodriguez-Aguayo C, Chavez-Reyes A, Lin YG, Carey MS, Nick AM, et al: c-Jun-NH2-kinase-1 inhibition leads to antitumor activity in ovarian cancer. Clin Cancer Res 16: 184-194, 2010.

29. Hayakawa J, Depatie C, Ohmichi M and Mercola D: The activation of c-Jun NH2-terminal kinase (JNK) by DNA-damaging agents serves to promote drug resistance via activating transcription factor 2 (ATF2)-dependent enhanced DNA repair. J Biol Chem 278: 20582-20592, 2003.

30. Hayakawa J, Mittal S, Wang Y, Korkmaz KS, Adamson E, English C, Ohmichi M, McClelland M and Mercola D: Identification of promoters bound by c-Jun/ATF2 during rapid large-scale gene activation following genotoxic stress. Mol Cell 16: 521-535, 2004.
31. Li QQ, Lee RX, Liang H, Wang G, Li JM, Zhong Y and Reed E: $\beta$-Elemene enhances susceptibility to cisplatin in resistant ovarian carcinoma cells via downregulation of ERCC-1 and XIAP and inactivation of JNK. Int J Oncol 43: 721-728, 2013.

32. Ohmichi M, Hayakawa J, Tasaka K, Kurachi H and Murata Y: Mechanisms of platinum drug resistance. Trends Pharmacol Sci 26: 113-116, 2005.

33. Potapova O, Haghighi A, Bost F, Liu C, Birrer MJ, Gjerset R and Mercola D: The Jun kinase/stress-activated protein kinase pathway functions to regulate DNA repair and inhibition of the pathway sensitizes tumor cells to cisplatin. J Biol Chem 272: 14041-14044, 1997.

34. Parekh H and Simpkins H: Cross-resistance and collateral sensitivity to natural product drugs in cisplatin-sensitive and -resistant rat lymphoma and human ovarian carcinoma cells. Cancer Chemother Pharmacol 37: 457-462, 1996.

35. Stordal B, Hamon M, McEneaney V, Roche S, Gillet JP, O'Leary JJ, Gottesman M and Clynes M: Resistance to paclitaxel in a cisplatin-resistant ovarian cancer cell line is mediated by P-glycoprotein. PLoS One 7: e40717, 2012.

36. Xu H, Choi SM, An CS, Min YD, Kim KC, Kim KJ and Choi $\mathrm{CH}$ : Concentration-dependent collateral sensitivity of cisplatin-resistant gastric cancer cell sublines. Biochem Biophys Res Commun 328: 618-622, 2005.

37. Yang H, Zou W, Li Y, Chen B and Xin X: Bridge linkage role played by CD98hc of anti-tumor drug resistance and cancer metastasis on cisplatin-resistant ovarian cancer cells. Cancer Biol Ther 6: 942-947, 2007.

38. Yang LY, Trujillo JM, Siciliano MJ, Kido Y, Siddik ZH and Su YZ: Distinct P-glycoprotein expression in two subclones simultaneously selected from a human colon carcinoma cell line by cis-diamminedichloroplatinum (II). Int J Cancer 53: 478-485, 1993.

39. Yang $\mathrm{X}$ and Pagé M: P-glycoprotein expression in ovarian cancer cell line following treatment with cisplatin. Oncol Res 7: 619-624, 1995

40. Comerford KM, Cummins EP and Taylor CT: c-Jun NH2-terminal kinase activation contributes to hypoxia-inducible factor 1alpha-dependent P-glycoprotein expression in hypoxia. Cancer Res 64: 9057-9061, 2004

41. Liu M, Li D, Aneja R, Joshi HC, Xie S, Zhang C and Zhou J: $\mathrm{PO}(2)$-dependent differential regulation of multidrug resistance 1 gene expression by the c-Jun NH2-terminal kinase pathway. J Biol Chem 282: 17581-17586, 2007.

42. Fitzgerald JB, Schoeberl B, Nielsen UB and Sorger PK: Systems biology and combination therapy in the quest for clinical efficacy. Nat Chem Biol 2: 458-466, 2006.

43. Lee MJ, Ye AS, Gardino AK, Heijink AM, Sorger PK, MacBeath G and Yaffe MB: Sequential application of anticancer drugs enhances cell death by rewiring apoptotic signaling networks. Cell 149: 780-794, 2012.

44. Morton SW, Lee MJ, Deng ZJ, Dreaden EC, Siouve E, Shopsowitz KE, Shah NJ, Yaffe MB and Hammond PT: A nanoparticle-based combination chemotherapy delivery system for enhanced tumor killing by dynamic rewiring of signaling pathways. Sci Signal 7: ra44, 2014. 